\title{
LA-UR-19-29768
}

Approved for public release; distribution is unlimited.

Title: Development of Computational Tools for Laser and Electron Beam Welding

Author(s): $\quad$ Patterson, Tate

Hochanadel, Joris E.

Lippold, John C.

Panton, Boyd E.

Tung, David C.

Johnson, Matthew Q.

Intended for: $\quad$ Present in Ohio State University welding engineering graduate student seminar.

Issued:

2019-09-26 
Disclaimer:

Los Alamos National Laboratory, an affirmative action/equal opportunity employer, is operated by Triad National Security, LLC for the National Nuclear Security Administration of U.S. Department of Energy under contract 89233218CNA000001. By approving this article, the publisher recognizes that the U.S. Government retains nonexclusive, royalty-free license to publish or reproduce the published form of this contribution, or to allow others to do so, for U.S. Government purposes. Los Alamos National Laboratory requests that the publisher identify this article as work performed under the auspices of the U.S. Department of Energy. Los Alamos National Laboratory strongly supports academic freedom and a researcher's right to publish; as an institution, however, the Laboratory does not endorse the viewpoint of a publication or guarantee its technical correctness. 


\section{Development of Computational Tools for Laser and Electron Beam Welding}

T. Patterson, J.E. Hochanadel, J.C. Lippold, B.E. Panton The Ohio State University

D.C. Tung, M.Q. Johnson

Los Alamos National Laboratory

30 September 2019 


\section{Background}

- Defense and aerospace applications commonly utilize laser beam welding (LBW) and electron beam welding (EBW) to join highly critical joints

Materials of interest include:

- 304L Stainless Steel

- Ti-6Al-4V

- Refractory Alloy

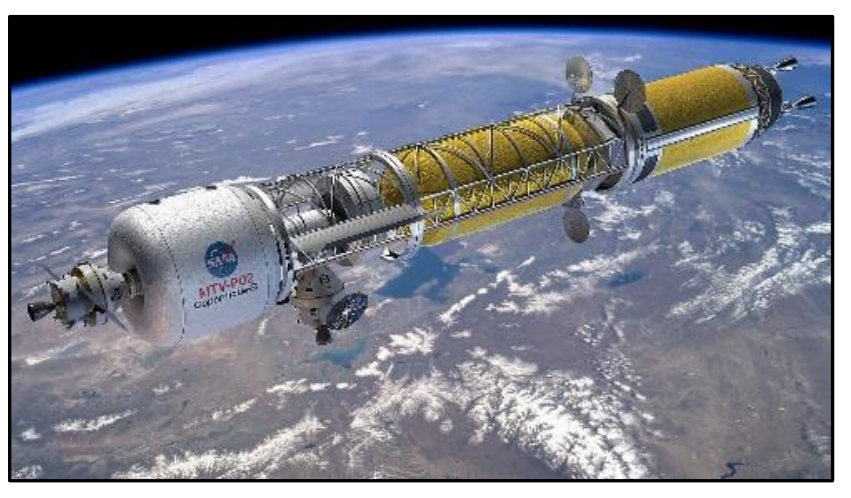

https://phys.org/news/2019-07-earth-mars-days-powernuclear.html

- Joint configurations are not always susceptible to postweld inspection 


\section{Background}

- Need to validate weld characteristics given parameter inputs

- Weld dimensions

- Penetration depth

- Keyhole width

- Cross-sectional area

- Thermal History

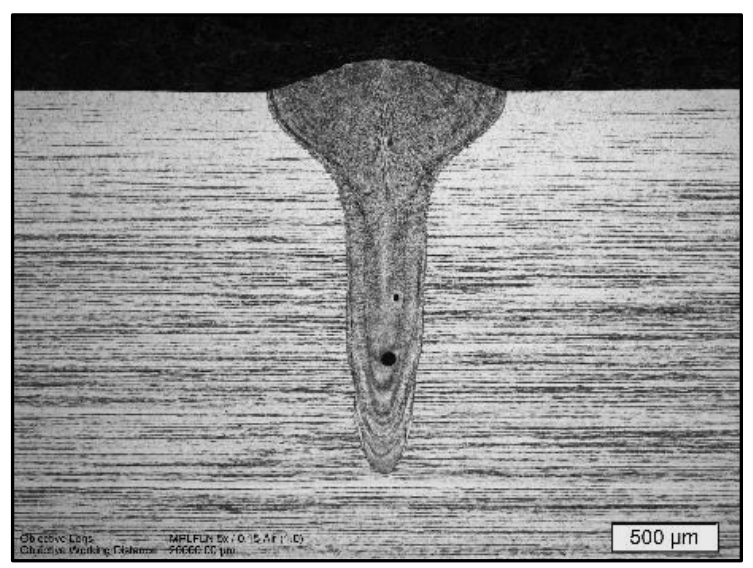

- Microstructural evolution

- How do process parameters influence weld geometry and microstructural formation

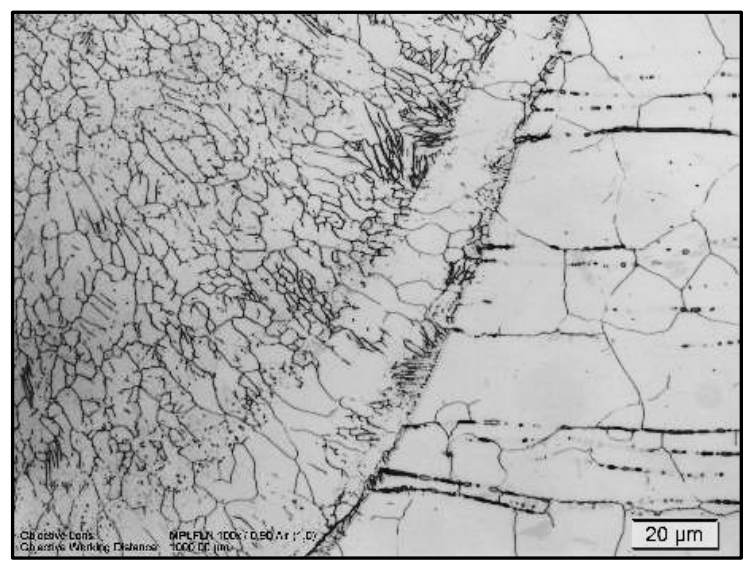




\section{Background}

\section{Process Parameters}

- Power

- Voltage and Current (EBW Only)

- Travel Speed

- Focus position

- Beam Diameter

- Beam Profile (intensity distribution)

Material Properties

- Density

- Thermal Conductivity

- Specific Heat

- Thermal Diffusivity
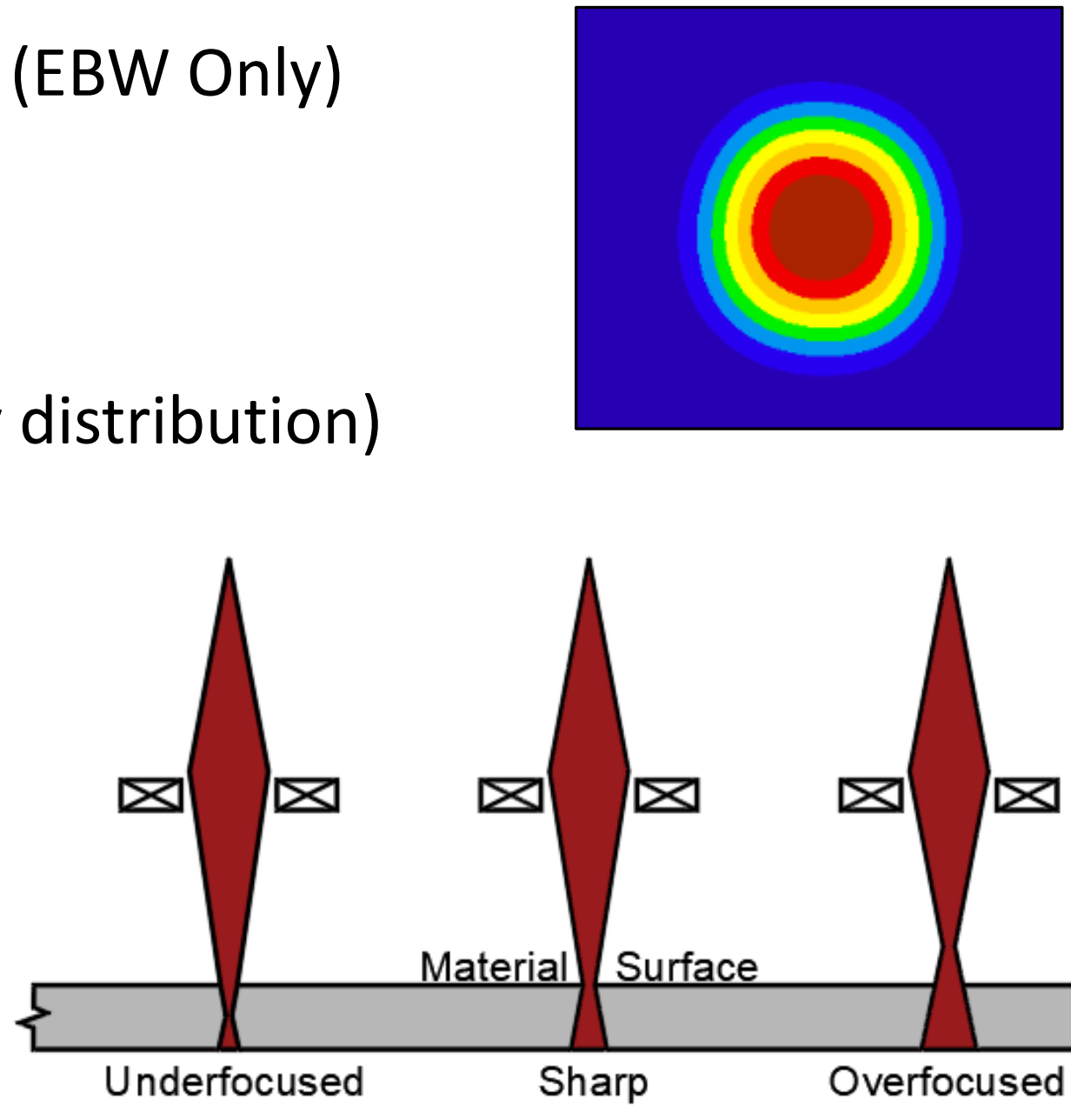

The OHIO STAYT UNIVERSTYY

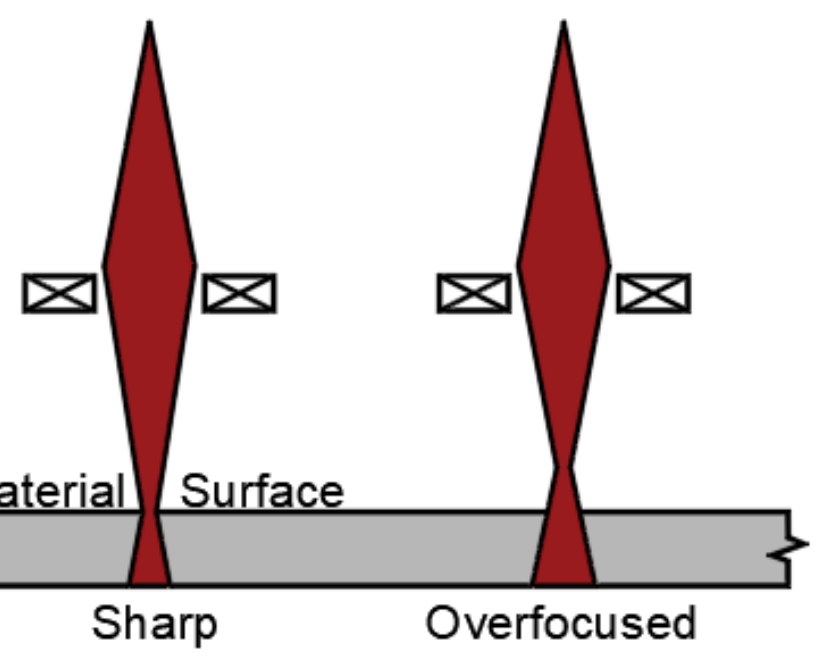




\section{Background}

\section{Beam Parameter Manipulation}

- Interaction Time and Specific Point Energy

$$
\begin{array}{cc}
t_{i}=d_{b} / v & {[s]} \\
\mathrm{SPE} & =\mathrm{P} * t_{i}
\end{array}
$$

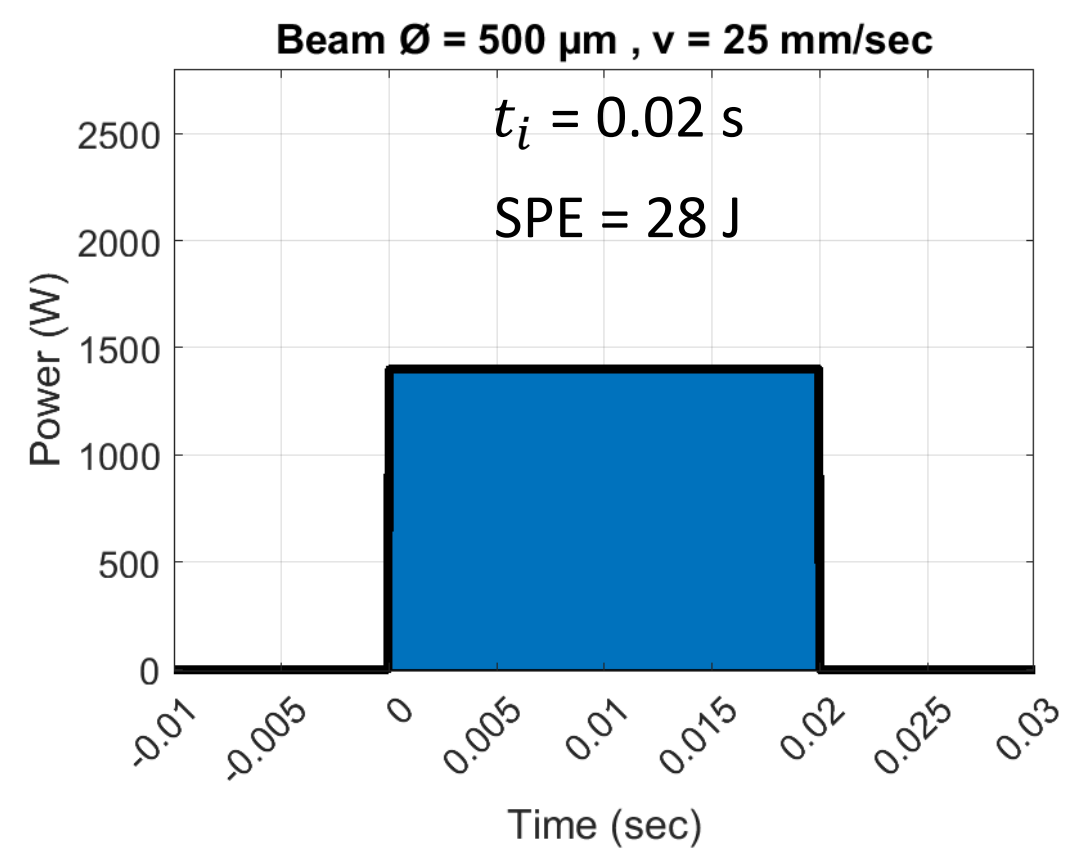

Where: $d_{b}=$ beam diameter $v=$ travel speed $P=$ beam power

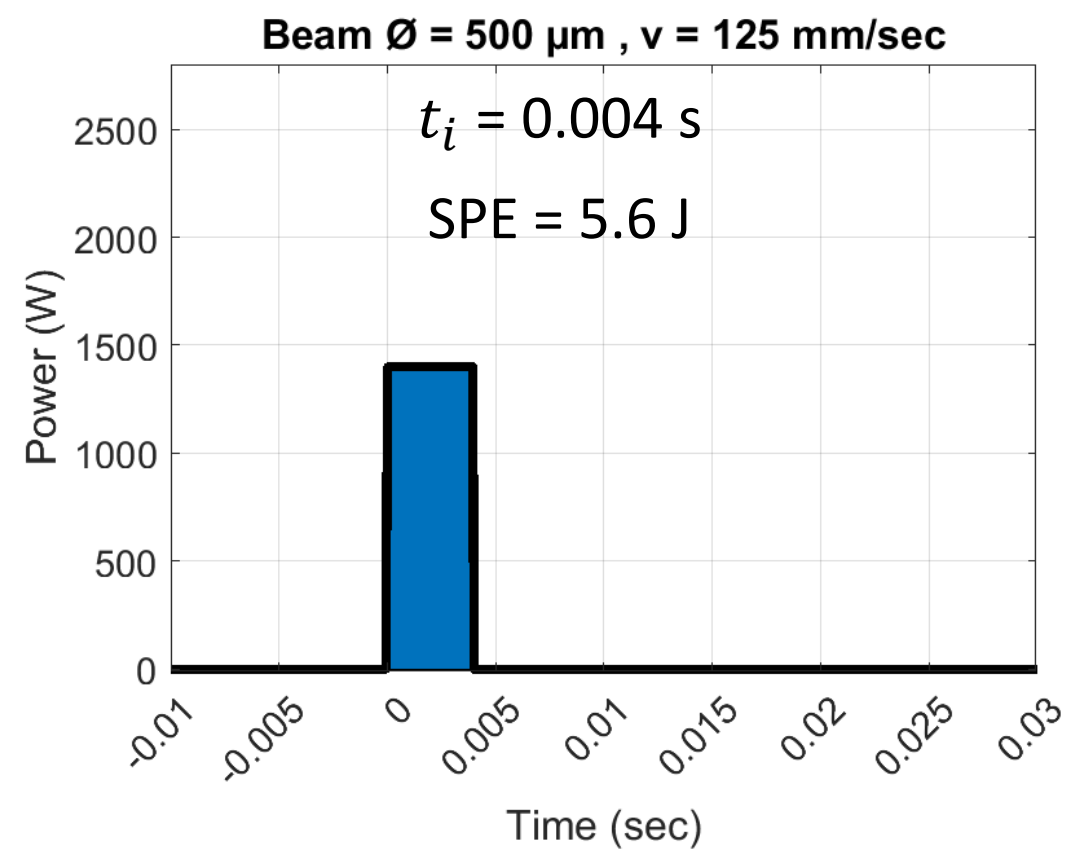




\section{Literature Review}

\section{Suder and Williams, 2012 [1]}

- Top-hat beam distribution

- YLR-8000 IPG laser

$$
\text { Beam diameter }=630 \mu \mathrm{m}
$$
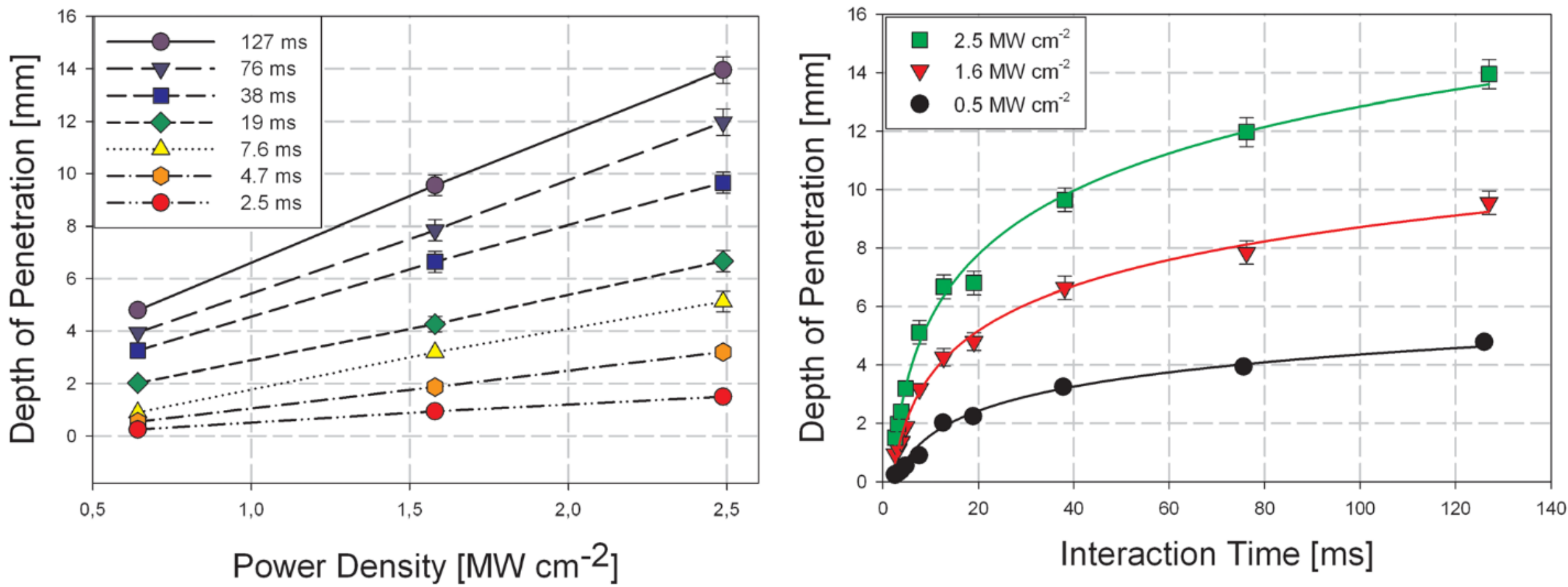


\section{Literature Review}

\section{Hann et. al, $2011[2,3]$}

- Incorporation of input parameters and material properties to predict penetration depths

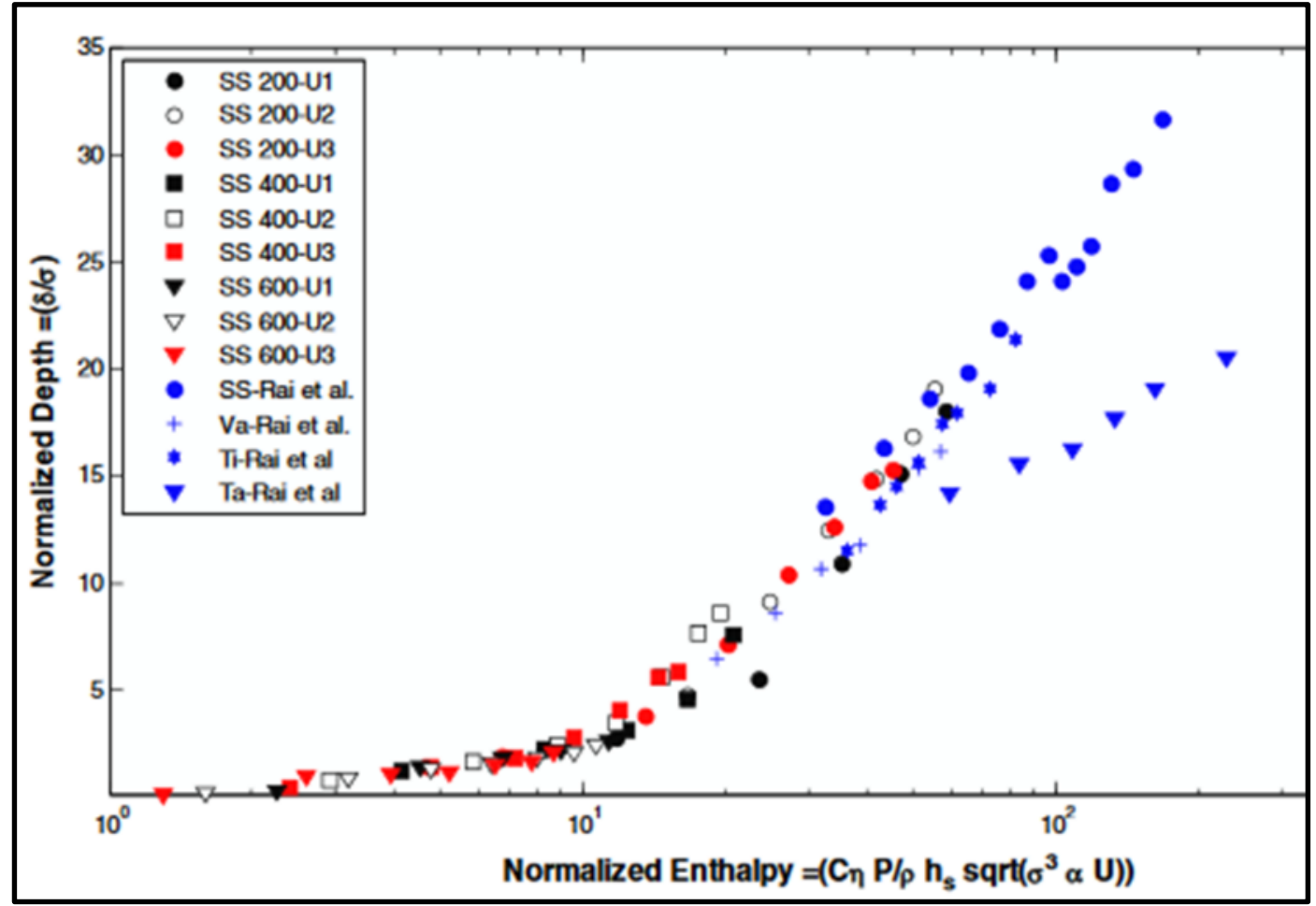

Dimensionless depth

$$
\begin{gathered}
\delta^{*}=f\left(B \frac{P}{\sqrt{\sigma^{3} U}}\right) \\
\delta^{*}=f\left(\frac{\Delta H}{h_{s}}\right)
\end{gathered}
$$

Where:

$\delta$ : depth of penetration

$\delta^{*}$ : normalized depth

$\boldsymbol{\sigma}:$ beam radius

B : normalization constant

P : power

$\mathbf{U}$ : travel speed

$\boldsymbol{\Delta H}$ : change in enthalpy

$\boldsymbol{h}_{\boldsymbol{s}}$ : enthalpy at melting 


\section{Literature Review}

\section{Lippold, 1994 [4]; Elmer et al., 1989 [5]}

- Influence of input parameters on solidification modes and microstructure

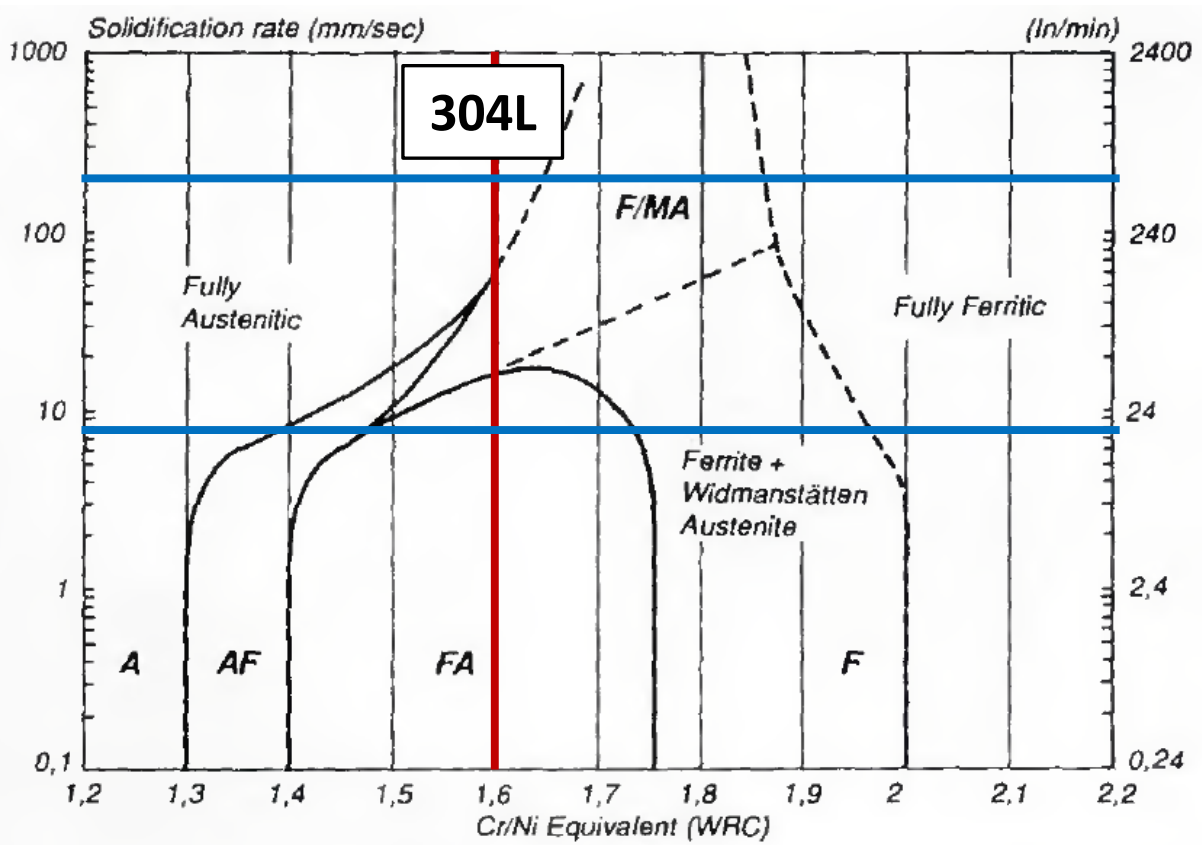

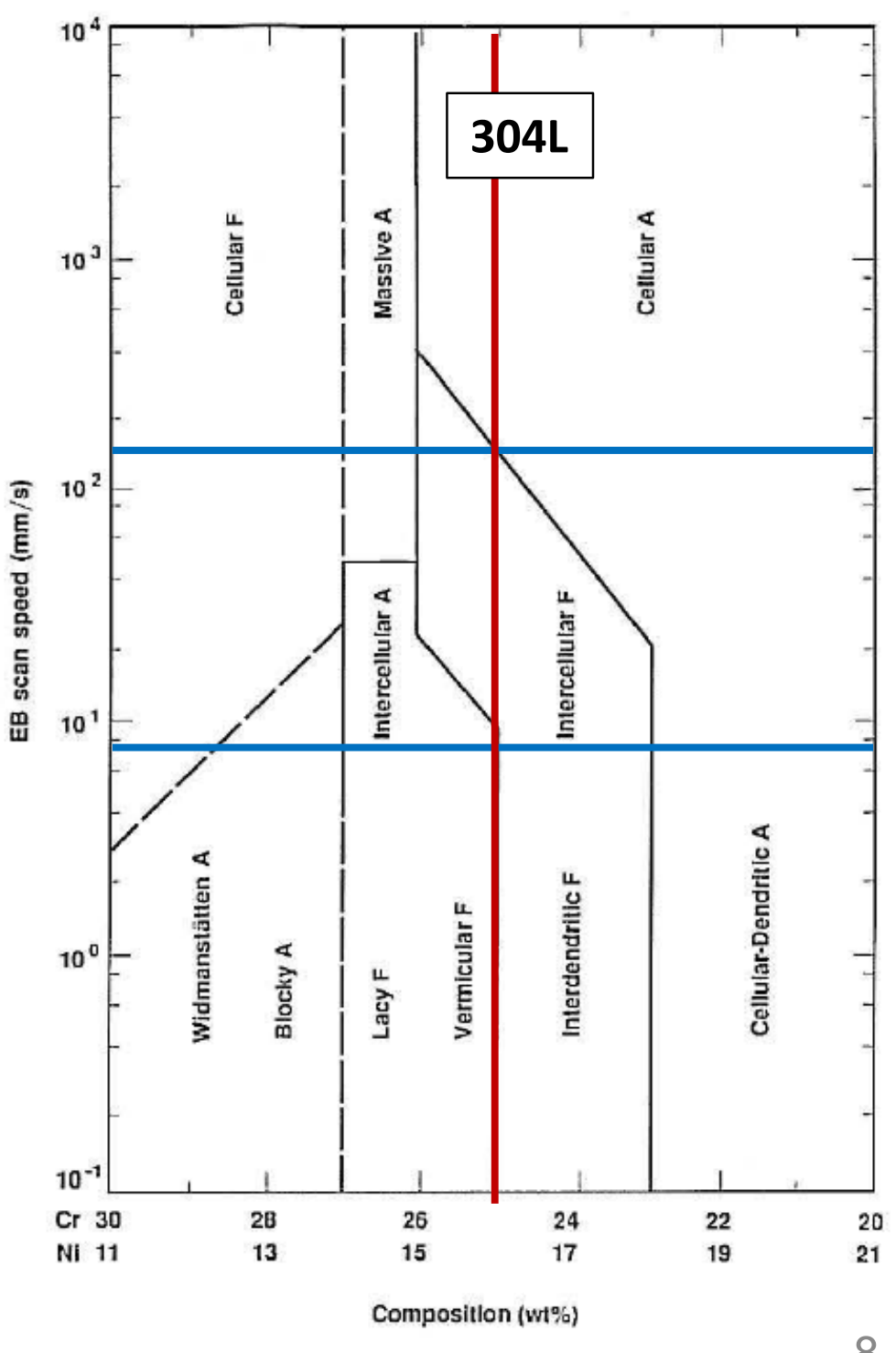

[5] 


\section{LBW Parameters}

\section{IPG YLS - 4000}

- $207.5 \pm 2 \mathrm{~mm}$ working distance

- $0.5 \mathrm{~mm}$ spot size

- $0^{\circ}$ beam angle

- Ar plume suppression 15 CFH (trailing)

- 304 S.S.

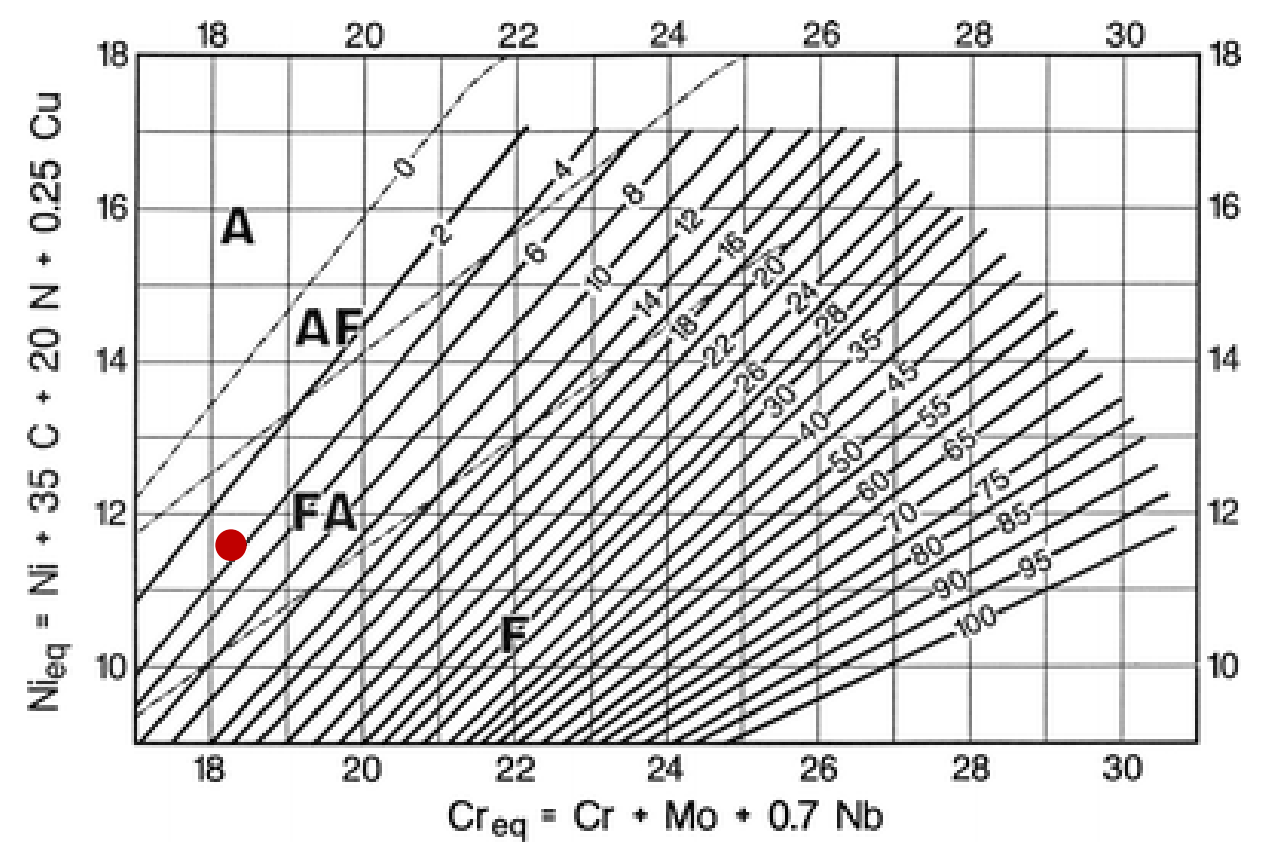

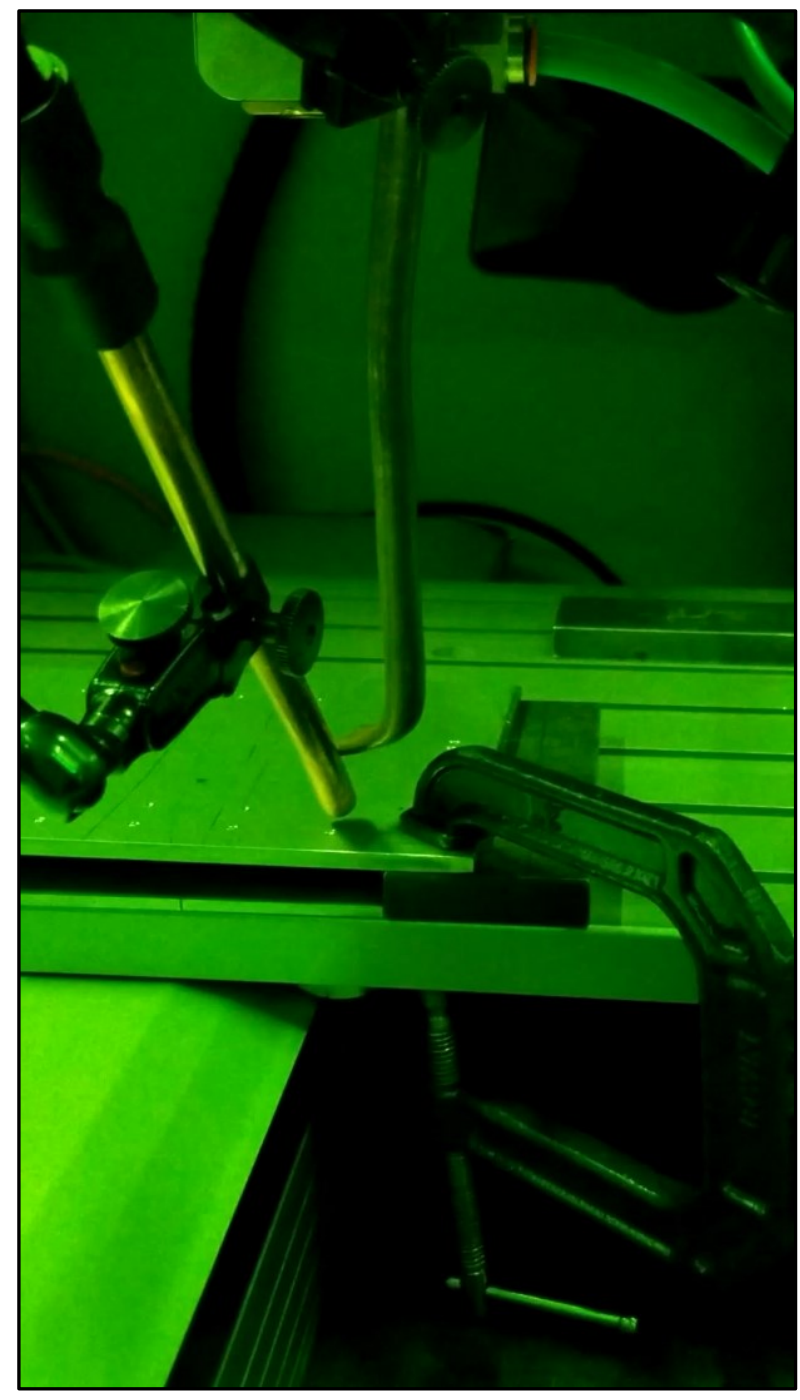

*slowed by $10 \mathrm{fps}$ 


\section{LBW Results on 304 S.S.}

\section{Travel Speed $=\mathbf{2 5} \mathrm{mm} / \mathrm{sec}$}

Interaction Time $\left(t_{i}\right)=20 \mathrm{msec}$
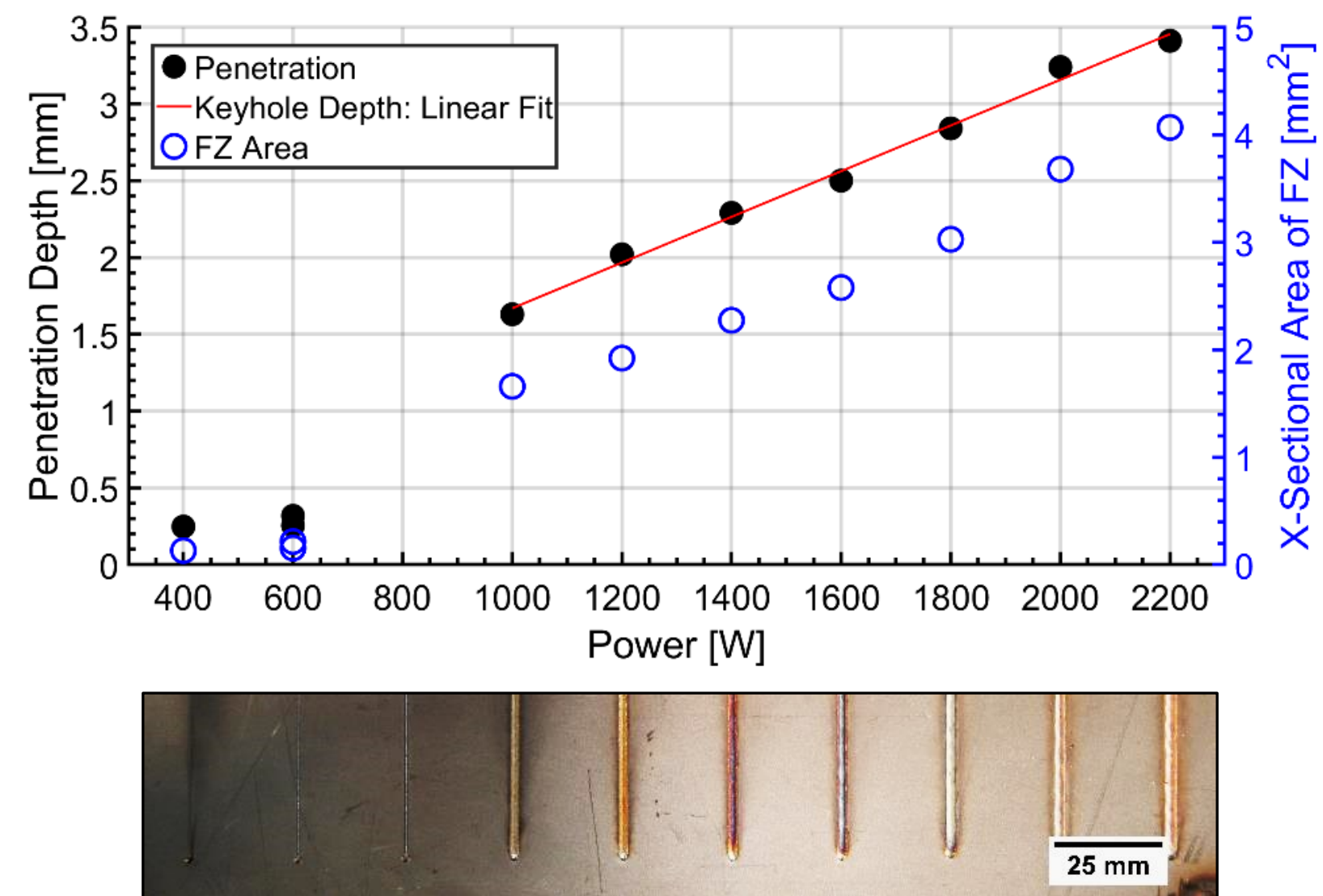

$P=600 W$ $200 \mu \mathrm{m}$

$P=1000 W$ $500 \mu \mathrm{m}$

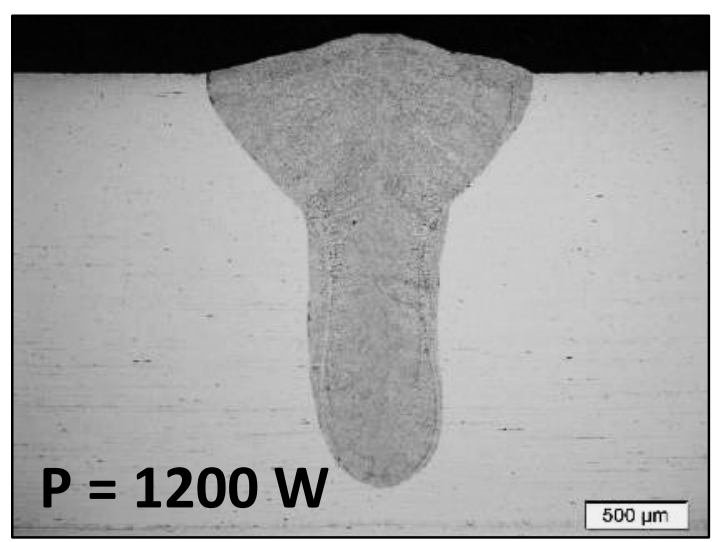




\section{LBW Results on 304 S.S.}

\section{Power $=1400 \mathrm{~W}$}

\section{Power Density $=7.1 \mathrm{~kW} / \mathrm{mm}^{2}$}
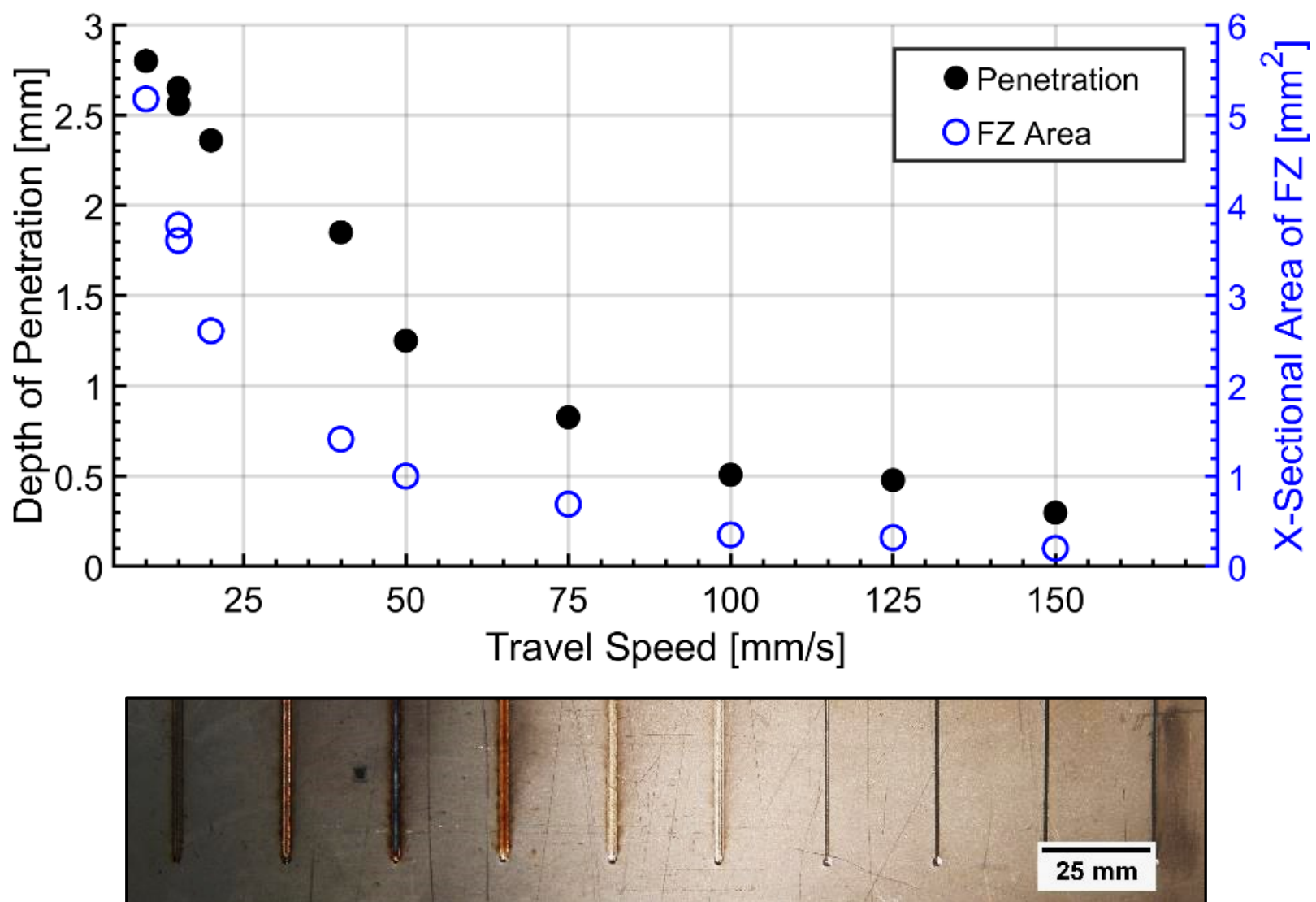
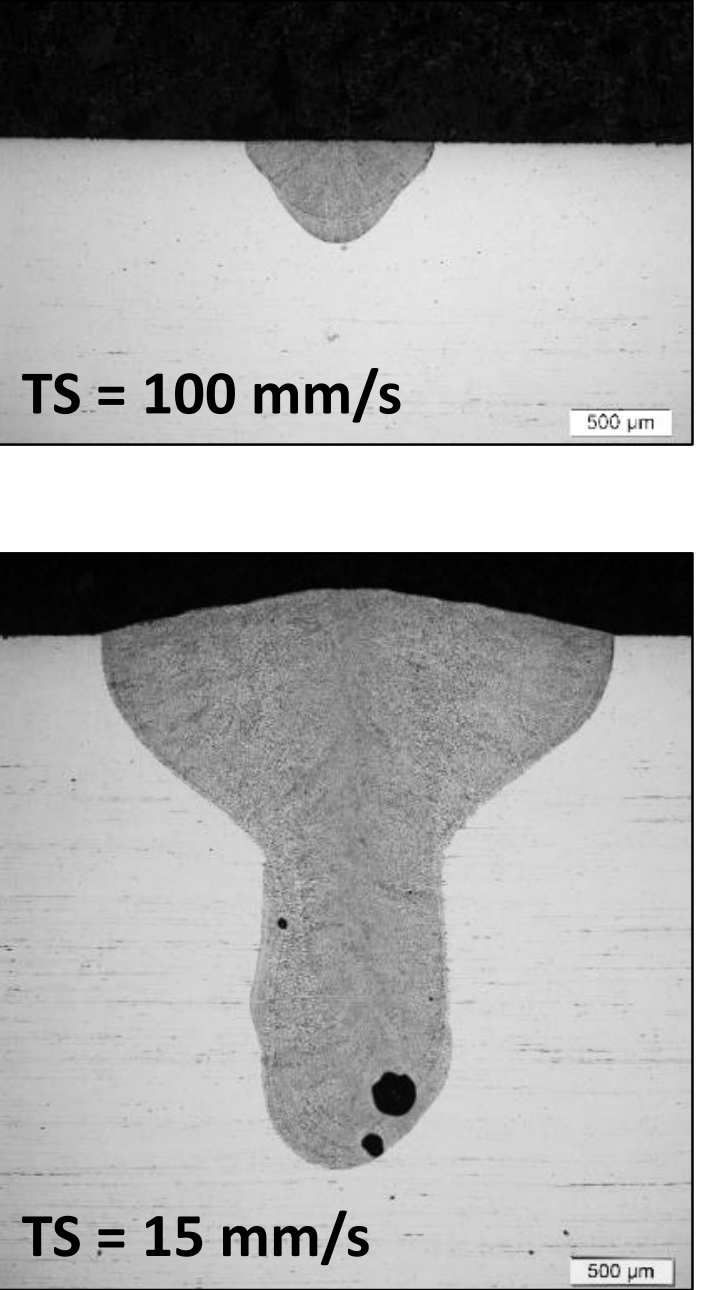


\section{LBW Results on 304 S.S.}

\section{SPE $=30 \mathrm{~J}$}

Energy Density $=153 \mathrm{~J} / \mathrm{mm}^{2}$
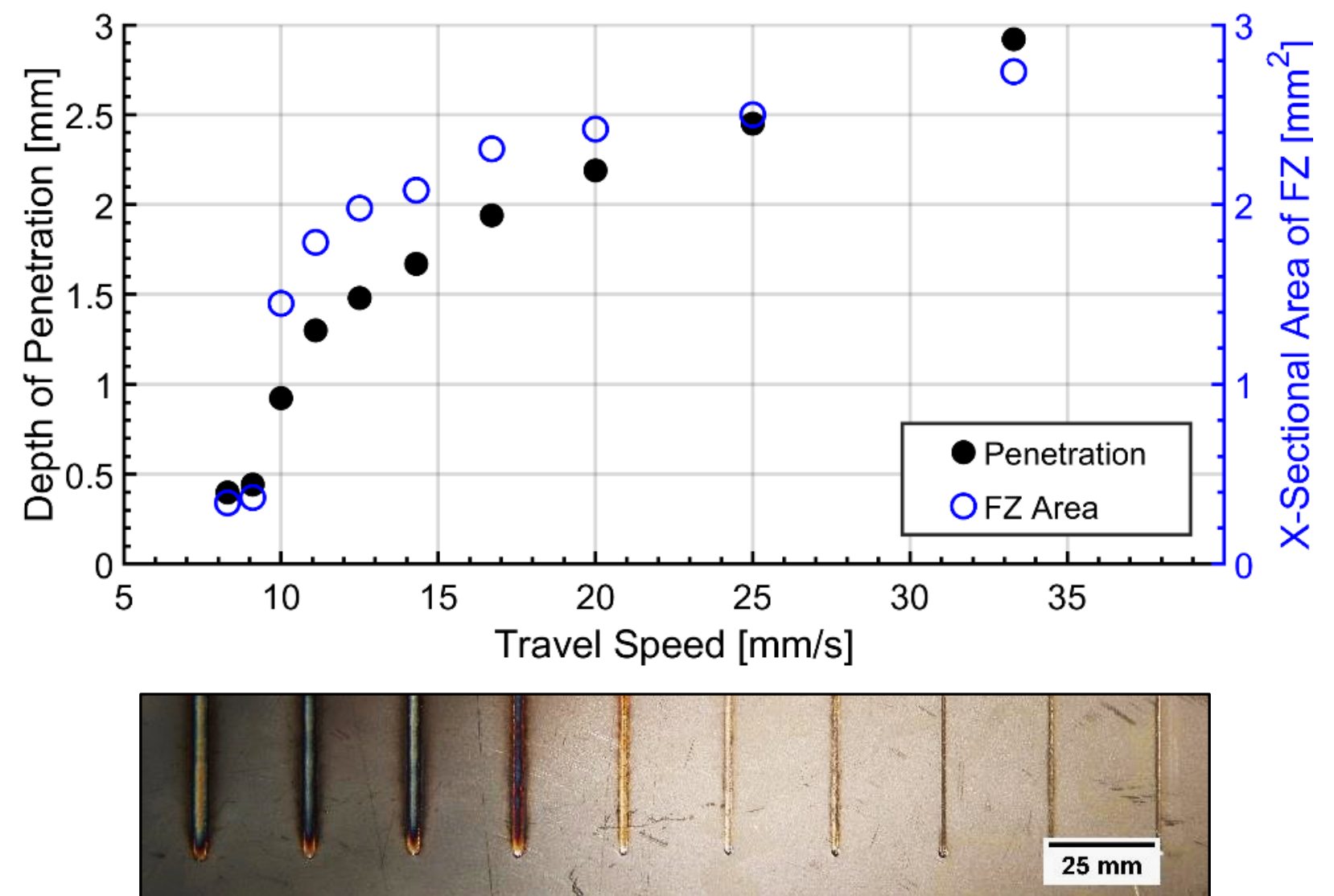

$P=545 W$

$200 \mu \mathrm{m}$
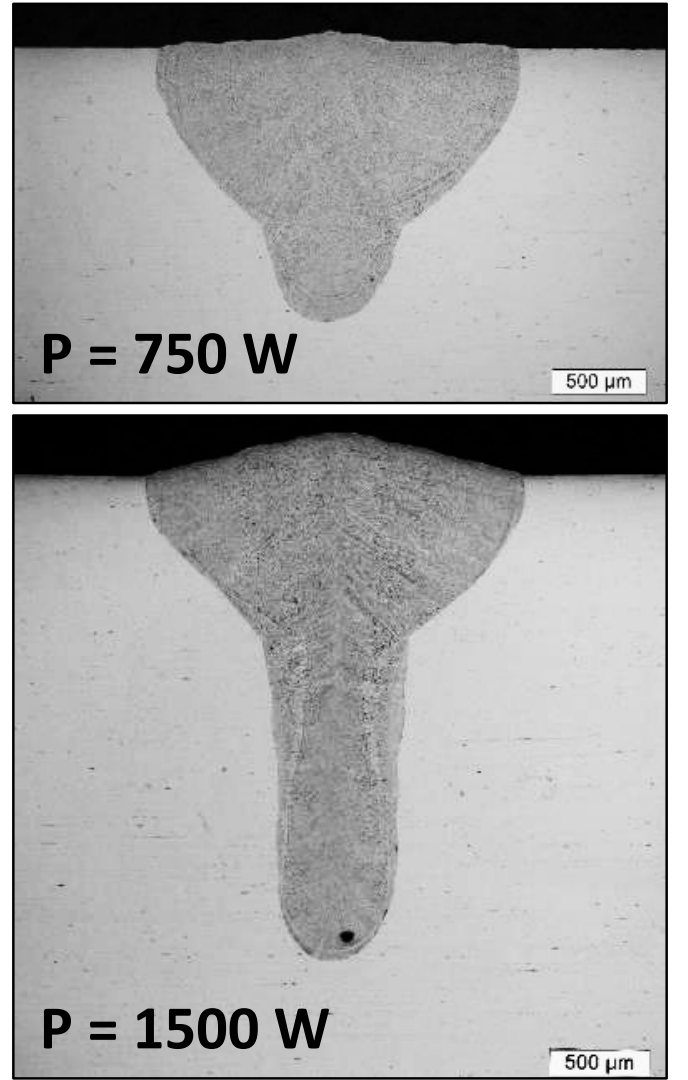


\section{LBW Results on 304 S.S.}

- Data Normalized Based on Hann et al. [3] Equations

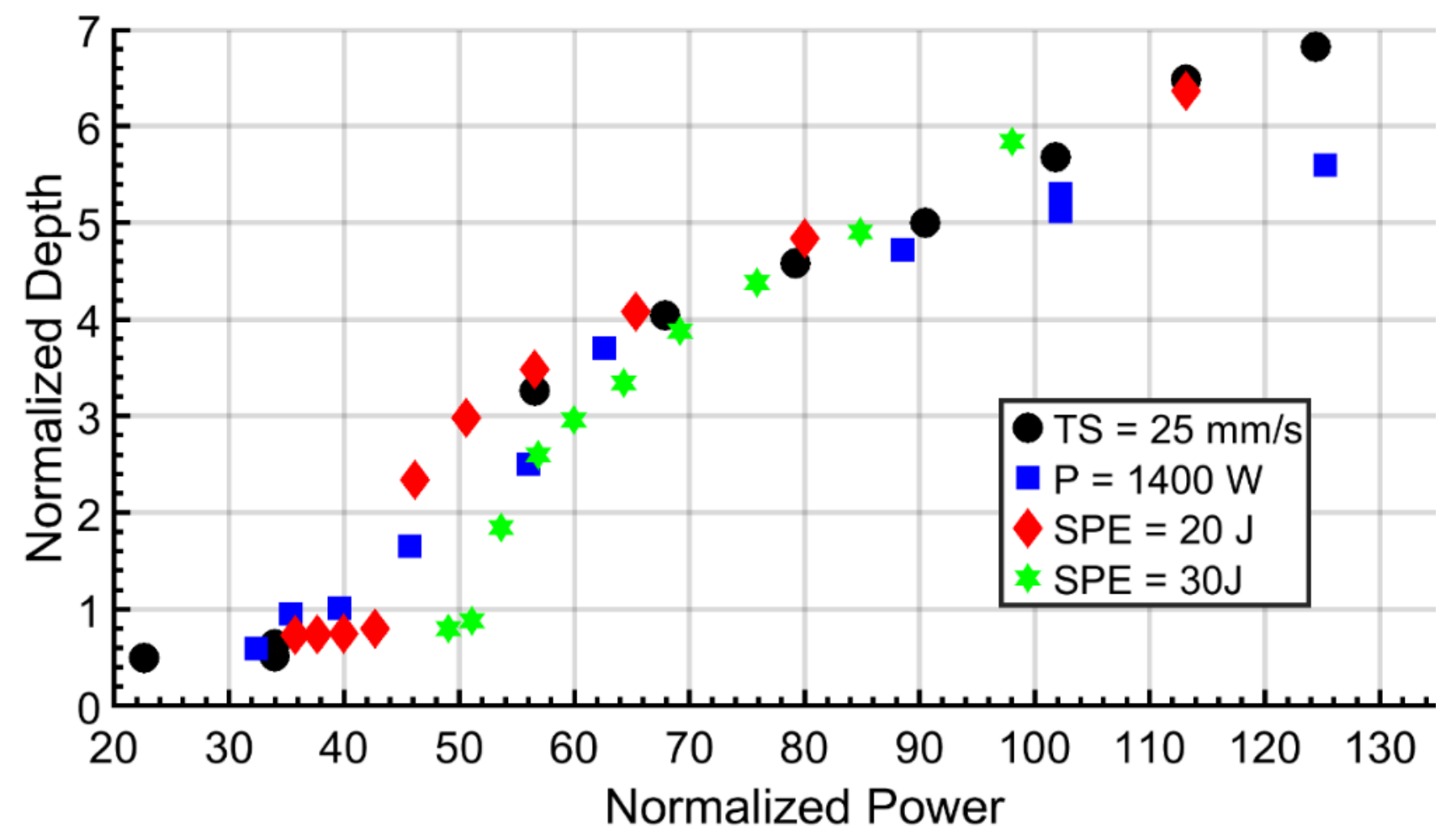

Normalized Depth $=$

$$
\left(\frac{\delta}{\sigma}\right)
$$

Normalized Power $=$

$$
\begin{gathered}
\frac{B * P}{\operatorname{sqrt}\left(\sigma^{3} U\right)} \\
B=1 \times 10^{-7} \frac{\mathrm{s}^{1 / 2}}{\mathrm{Jm}^{2}} \\
* \text { For } 304 \text { S.S. }
\end{gathered}
$$




\section{LBW Results: Microstructure Evolution}
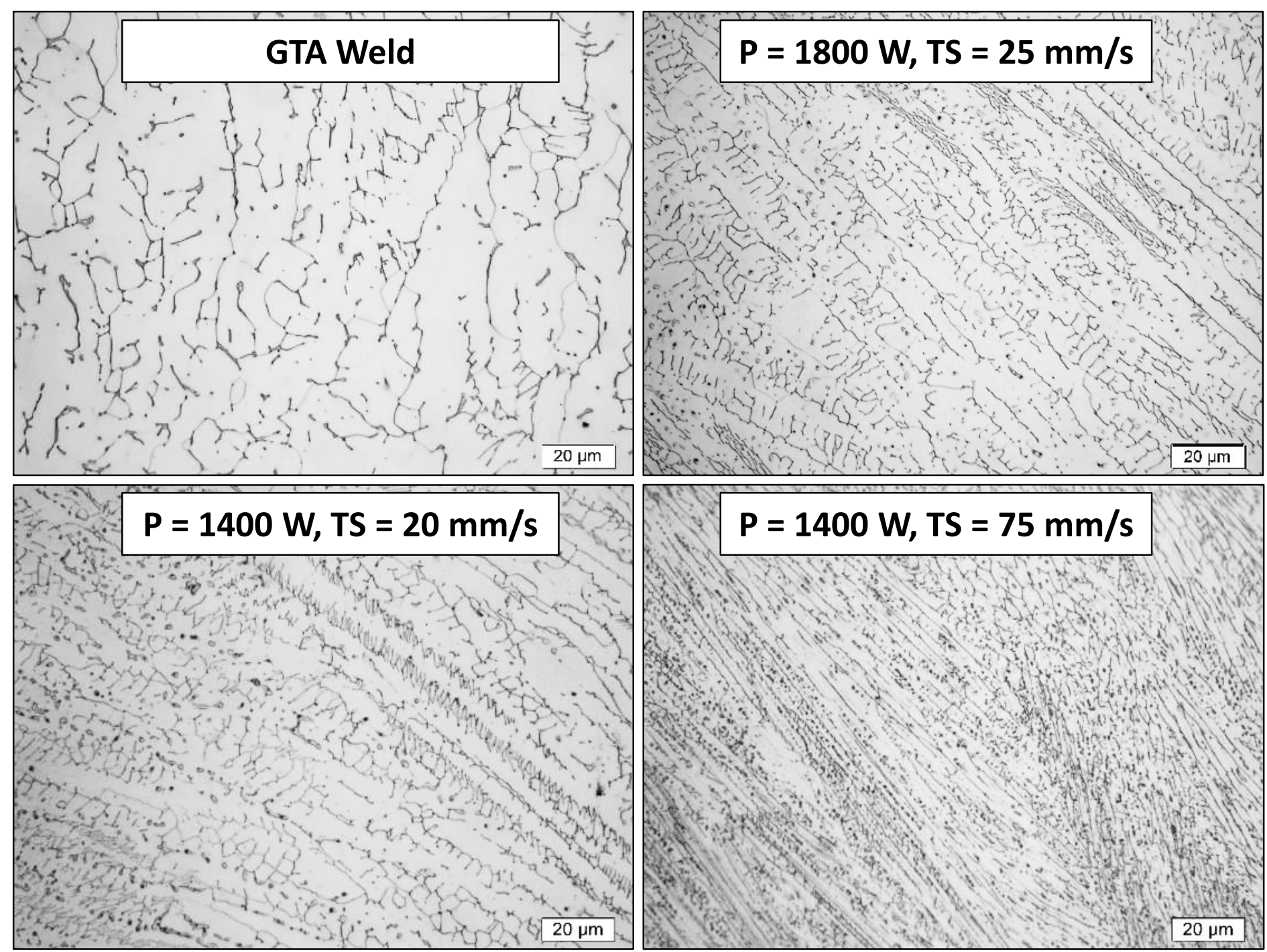


\section{EBW Parameters}

\section{Pro-Beam K10}

- high voltage system

- $\approx 200 \mathrm{~mm}$ gun to work distance

- $0.5 \mathrm{~mm}$ beam diameter

- high vacuum (< $1 \times 10^{-4}$ torr)

- 304L S.S.
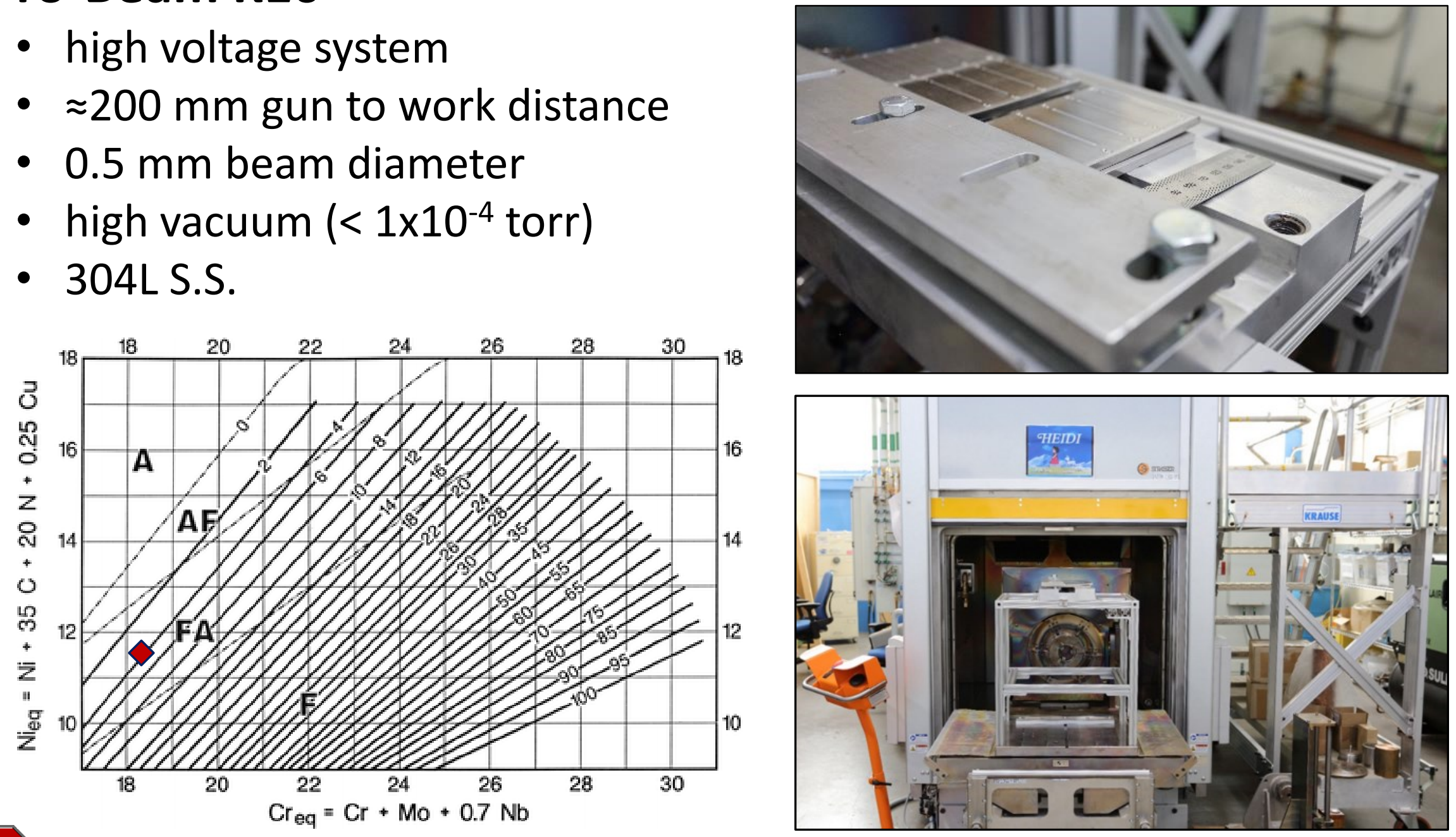

THE OHIO STATE UNIVERSITY 


\section{EBW Results on 304L S.S.}

\section{Constant Voltage $=100 \mathrm{kV}$}

\section{Constant Travel Speed $=\mathbf{2 5} \mathrm{mm} / \mathrm{s}$}
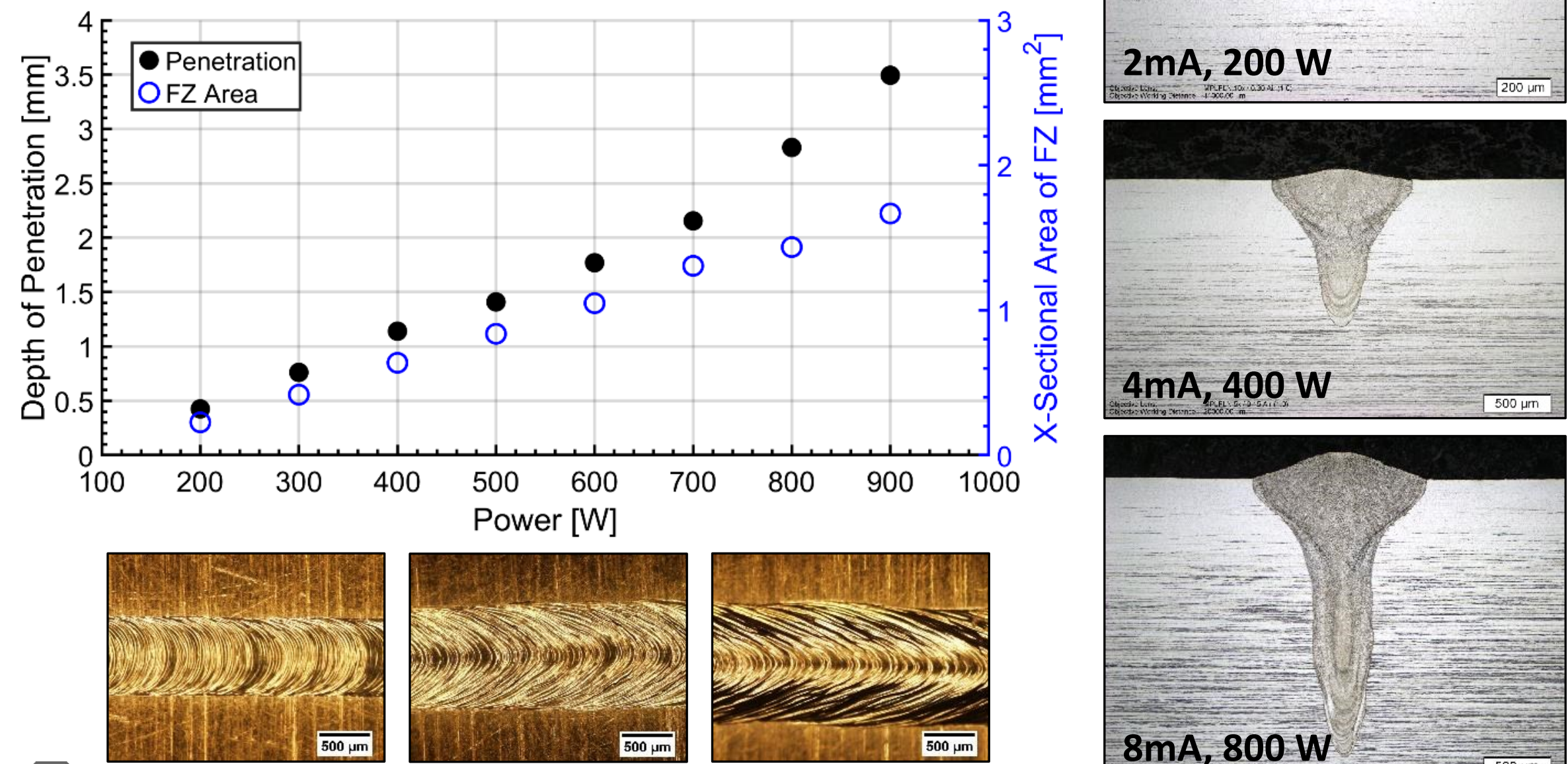

ThE OHIO STATE UNIVERSITY

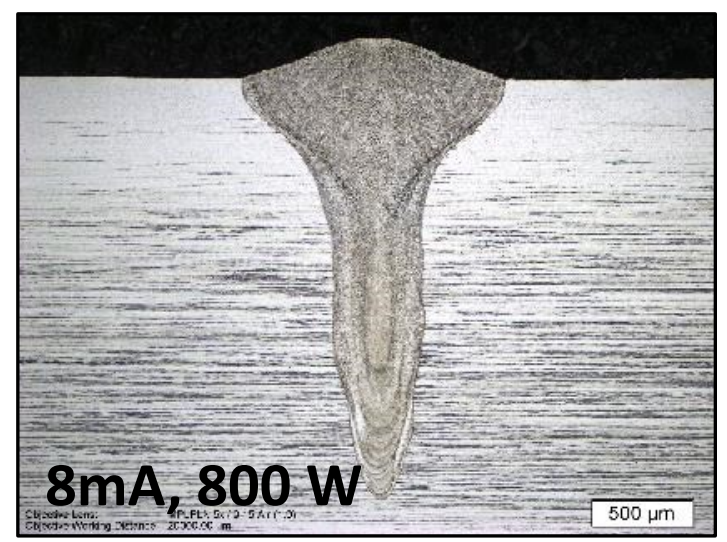




\section{EBW Results on 304L S.S.}

\section{Constant Current $=6 \mathrm{~mA}$}

Constant Travel Speed $=25 \mathrm{~mm} / \mathrm{s}$
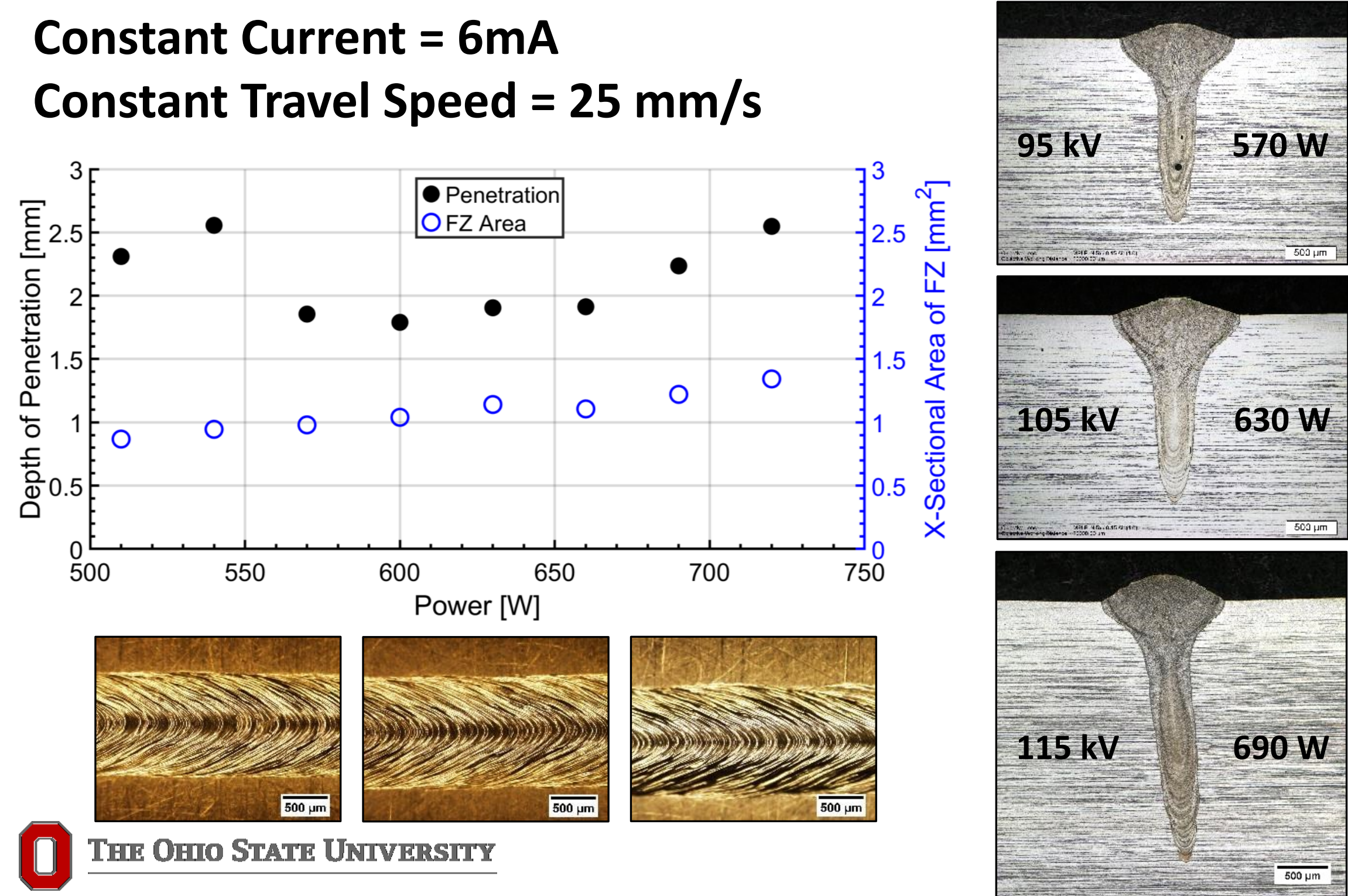


\section{EBW Results on 304L S.S.}

\section{Constant Current $=6 \mathrm{~mA}$}

\section{Constant Voltage $=100 \mathrm{kV}$}
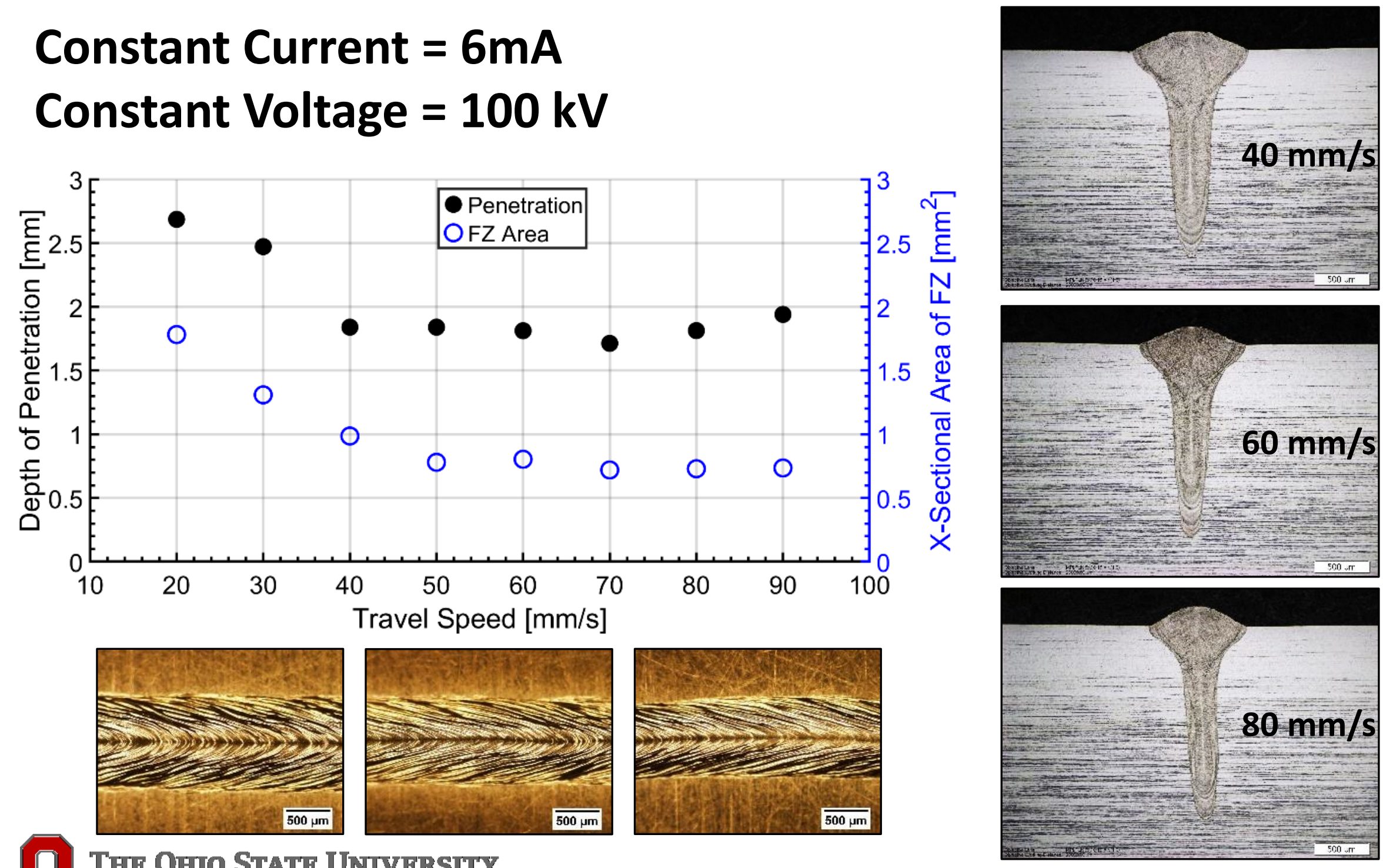

THE OHIO STATE UNIVERSITY 


\section{Conclusions}

\section{LBW}

- Constant travel speed and beam diameter produced a linear increase in keyhole penetration depth

- Constant power showed a non-linear decrease in penetration depth

- Travel speed has largest influence on microstructure variations in 304 S.S.

\section{EBW}

- Increasing current showed an increase in penetration depth

- Increasing voltage showed no correlation with penetration

- Penetration decreased with constant power until travel speeds were in excess of $60 \mathrm{~mm} / \mathrm{s}$ 


\section{Future Work}

- Perform in-depth characterization of the weld metal microstructures

- Analyze solidification modes, phase fractions, and dendrite arm spacing

- Measure the beam profile of the laser

- Complete laser welds on Ti-6Al-4V and 304L

- Understand the data to produce a predictive tool to determine weld geometries based on input parameters and material properties

- Produce microstructural maps for solidification profiles and phase fractions 


\section{References}

[1] W. J. Suder and S. W. Williams, "Investigation of the effects of basic laser material interaction parameters in laser welding," J. Laser Appl., vol. 24, no. 3, p. 032009, 2012.

[2] D. B. Hann, J. lammi, and J. Folkes, "A simple methodology for predicting laser-weld properties from material and laser parameters," J. Phys. D. Appl. Phys., vol. 44, no. 44, 2011.

[3] D. B. Hann, J. lammi, and J. Folkes, "Keyholing or Conduction - Prediction of Laser Penetration Depth," Proc. 36th Int. MATADOR Conf., vol. 22, no. 0, pp. 275-278, 2010.

[4] J. C. Lippold, "Solidification Behavior and Cracking Susceptibility of Pulsed-Laser Welds in Austenitic Stainless Steels A shift in solidification behavior under rapid solidification conditions promotes an increase in cracking susceptibility," Weld. J., vol. 73, no. 6, pp. 129-139, 1994.

[5] J. W. Elmer, S. M. Allen, and T. W. Eagar, "Microstructural Development during Solidification of Stainless Steel Alloys," Metall. Trans. A, vol. 20A, no. October, pp. 2117-2131, 1989.

[6] D. J. Kotecki and T. A. Siewart, "WRC-1992 Constitution Diagram for Stainless Steel Weld Metals : A Modification of the WRC-1988 Diagram," Weld. J., no. May, pp. 171-178, 1992. 


\section{Acknowledgments}

Manufacturing and Materials Joining Innovation Center

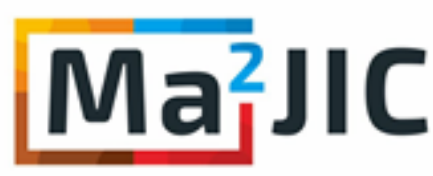

MANUFACTURING \& MATERIALS

JOINING INNOVATION CENTER

Los Alamos National Laboratory

Brett Krueger, Rich Goodbody, Diego Ortiz,

Alex Lloyd, Dr. Paul Burgardt

IPG Photonics

Kevin Arnold, Eric Stiles, Erin Lalinsky 


\section{Extra Slides}

